\title{
Risk Factors Associated with Postoperative Discomfort After Ambulatory Strabismus Surgery Under General Anesthesia
}

This article was published in the following Dove Press journal: Journal of Pain Research

\author{
Jingxia Huang ${ }^{1, *}$ \\ Jing Lin $\mathbb{1 D}^{2, *}$ \\ Yun Xiong ${ }^{2}$ \\ Zhonghao Wang ${ }^{2}$ \\ Yanling Zhu (D)' \\ Huijing $\mathrm{Ye}^{3}$ \\ Wenjun Guo'
}

'Department of Anesthesia, State Key Laboratory of Ophthalmology, Zhongshan Ophthalmic Center, Sun Yat-sen University, Guangzhou, People's Republic of China;

${ }^{2}$ Department of Strabismus and Amblyopia, State Key Laboratory of Ophthalmology, Zhongshan Ophthalmic Center, Sun Yatsen University, Guangzhou, People's Republic of China; ${ }^{3}$ Department of Orbital Disease and Ocular Oncology, State Key Laboratory of Ophthalmology, Zhongshan Ophthalmic Center, Sun Yat-sen University, Guangzhou, People's Republic of China

*These authors contributed equally to this work

Correspondence: Wenjun Guo State Key Laboratory of Ophthalmology, Zhongshan Ophthalmic Center, Sun Yat-sen University, 54 South Xianlie Road, Guangzhou 510060, People's Republic of China

Tel +86 208733 I548

Fax +862087334825

Email 402385889@qq.com
Purpose: The aim of this study was to evaluate postoperative discomfort in patients undergoing ambulatory strabismus under general anesthesia, and to identify risk factors associated with the discomfort.

Patients and Methods: A cross-sectional study was conducted among 210 consecutive patients undergoing ambulatory strabismus under general anesthesia. Postoperative discomfort including nausea, vomiting, dizziness, and headache was recorded and quantified. Univariable and multivariable logistic regression were performed to detect the risk factors associated with postoperative discomfort.

Results: Of 210 participants, 199 (94.76\%) patients experienced mild discomfort after ambulatory strabismus surgery under general anesthesia, and 31 (14.76\%), 11 (5.24\%), 60 (28.57\%), 23 (10.95\%) patients suffered from nausea, vomiting, dizziness, and headache, respectively. A multivariate analysis indicated that female sex, the surgery on inferior rectus, and the surgery on inferior oblique were the independent risk factors for postoperative vomiting while, mild anxiety was the independent risk factor for postoperative dizziness.

Conclusion: Patients undergoing ambulatory strabismus surgery tended to experience postoperative nausea and dizziness. Female sex, the surgery on inferior rectus, mild anxiety, and the surgery on inferior oblique were the independent predictors of postoperative discomfort. Keywords: postoperative nausea and vomiting, dizziness, strabismus surgery, ambulatory surgery, related factors

\section{Introduction}

Ambulatory surgery has been introduced in many countries in recent decades, because of advantages of reducing surgical waiting times, overcoming bedshortage crises in hospitals, reducing health-care costs, and increasing patient satisfaction. ${ }^{1}$ Now, $65-70 \%$ of all operations in the United States are performed on an ambulatory basis. ${ }^{2}$ Recently, with the development of surgical and anesthesia techniques, the use of ambulatory surgery for strabismus has grown rapidly.

Ophthalmic surgery is widely believed to cause little or no postoperative pain for less extensive trauma compared to other types of surgery. ${ }^{3}$ Several studies have reported that adult patients complain of minimal pain after strabismus surgery. ${ }^{4,5}$ However, patient preference for a postoperative treatment does not seem to be based only on pain experience. ${ }^{6}$ Discomfort, which was defined as "sensation other than pain", and included nausea, vomiting, dizziness, and headache, may play an important role. ${ }^{7}$ Furthermore, postoperative nausea and vomiting (PONV) are the 
most common complications after strabismus surgery under general anesthesia, with an incidence of $37-80 \%{ }^{8,9}$ The mechanism for the high rate of PONV following strabismus surgery has not been clearly defined, but appears to involve several factors, such as oculoemetic reflex, an optokinetic imbalance, and a disturbance of visual axes. ${ }^{10}$ Meanwhile, PONV are the commonest reasons for delaying discharge and unplanned admissions after ambulatory surgery. Thus, minimizing postoperative discomfort, especially PONV, would be crucial part of perioperative management of patients with ambulatory strabismus surgery.

Although postoperative discomfort symptoms are minor complications after strabismus surgery, they have a major impact on the quality of recovery in ambulatory strabismus surgery. Therefore, we conducted a cross-sectional study on factors associated with postoperative discomfort in 210 consecutive patients during the first 24 hours after ambulatory strabismus surgery with general anesthesia.

\section{Methods}

\section{Patients}

Consecutive patients, who underwent ambulatory strabismus surgery under general anesthesia, were enrolled into the study from July to September 2019, and were assessed for discomfort after surgery. The project was approved by the Ethics Committee of Zhongshan Ophthalmic Center, Sun Yat-sen University (approval registration number: 2019KYPJ056), and was registered at the Chinese Clinical Trial Registry: www.chictr.org.cn (No. ChiCTR -1900023799). This study was conducted in accordance with the Declaration of Helsinki. Each patient signed a written informed consent for participation in the study, and a parent or legal guardian provided written informed consent for patients under the age of 18 years.

\section{Inclusion and Exclusion Criteria}

Patients were screened before surgery. Adult patients (age 16 to 65 years) who underwent ambulatory strabismus surgery under general anesthesia were included in the study. The exclusion criteria were mental illness, inability to communicate (including impairment of sensorium) and refusal to participate.

\section{Protocol of Anesthesia}

No premedication was given. Upon arrival in the operating room, all patients were routinely monitored with the electrocardiogram, heart rate (HR), noninvasive blood pressure
(NIBP), pulse oxygen saturation level $\left(\mathrm{SpO}_{2}\right)$, and end-tidal carbon dioxide $\left(\mathrm{EtCO}_{2}\right)$. Anesthesia was induced by propofol $(1.5-2.5 \mathrm{mg} / \mathrm{kg})$ and fentanyl $(1-2 \mu \mathrm{g} / \mathrm{kg})$, and a laryngeal mask airway (LMA) was inserted. The maintenance of anesthesia was achieved using propofol $(6 \mathrm{mg} / \mathrm{kg} / \mathrm{h})$ and remifentanil $(0.15-0.2 \mu \mathrm{g} / \mathrm{kg} / \mathrm{min})$. Flurbiprofen axetil or nalbuphine were used intraoperatively to provide analgesia. Meanwhile, 5- $\mathrm{HT}_{3}$ receptor antagonist (dolasetron or tropisetron) combined with dexamethasone were administered intraoperatively as prevention for PONV. The operation was all conducted by a senior attending or associate chief strabismus surgeon who had performed more than 300 operations.

\section{Study Protocol}

A day before surgery, patients filled out the Self-rating Anxiety Scale (SAS, SAS score $<50=$ no anxiety, 50-59= mild anxiety, $61-70=$ moderate anxiety and $>70=$ severe anxiety), ${ }^{11,12}$ and patient sociodemographic characteristics were collected, including age, gender, body mass index (BMI), marital status, and education level. The other collected data included: smoking and drinking status, medical history of analgesics and sedative, history of prior surgery on the operative eye or the non-operative eye, preoperative American Society of Anesthesiologists (ASA) Physical Status.

After surgery, discomfort scores, antiemetic medication, and surgical and anesthesia data were recorded. The patients were asked to grade their discomfort using numerical rating scale (NRS) ranging from 0 to $10(0=$ no discomfort, $1-3=$ mild discomfort, $4-6=$ moderate discomfort, $7-10=$ severe discomfort). This scale has been confirmed to be sensitive and reliable. ${ }^{7,13}$ The degree of postoperative discomfort was measured $0,2,6,10$, and 24 hours after postanesthesia care unit (PACU). Discomfort included nausea, vomiting, dizziness, and headache, within 24 hours after PACU. The data about nausea, vomiting, dizziness, and headache were recorded.

\section{Statistical Analysis}

Data were presented as mean and standard deviation (SD) for normally distributed continuous data, median and interquartile range (IQR) for continuous data without normal distribution, and number (\%) for categorical data. Factors associated with nausea, vomiting, dizziness, headache were investigated using a logistic regression model. Candidate variables with a $\mathrm{p}$ value $<0.2$ on univariate analysis were included in multivariable model. $P<0.05$ was considered statistical significance. SPSS 24.0 (SPSS Inc., Chicago, IL, USA) was used for statistical analysis. 


\section{Results}

\section{Patients Characteristics Data}

A total of 210 consecutive patients were enrolled in this study, 102 patients were male and 108 were female. The median age was 27.0 (IQR, 22.0-33.3) years, and the median body mass index (BMI) was 21.2 (IQR, 19.4-23.4). Most patients had an ASA status of class $1(\mathrm{~N}=170,80.95 \%)$. Only five patients had mild anxiety (SAS score 50-59) (Table 1).

\section{Surgical and Anesthesia Data}

Most patients had a duration of operation $\leq 30 \mathrm{~min}$ $(\mathrm{N}=151,71.9 \%)$, a duration of anesthesia $\leq 60 \mathrm{~min}$ $(\mathrm{N}=160,76.19 \%)$, monocular surgery $(\mathrm{N}=152$, $72.38 \%)$, the surgery on medial rectus $(\mathrm{N}=164$, $78.10 \%)$ and lateral rectus $(\mathrm{N}=185,88.10 \%)$. Nalbuphine was given to $126(60 \%)$ patients; flurbiprofen axetil was given to $71(33.81 \%)$ patients. Most patients received a combination of anti-emetics, such as dolasetron and dexamethasone, or tropisetron and dexamethasone $(\mathrm{N}=203,96.67 \%)$ (Table 2).

Table I Characteristics of the Participants $(N=210)$

\begin{tabular}{|c|c|c|}
\hline Characteristics & $\mathbf{N}$ & Statistics \\
\hline Age, years, median (IQR) & 210 & $27.00(22.00-33.30)$ \\
\hline Female sex, n (\%) & 210 & $108(5 \mathrm{I} .43)$ \\
\hline BMI, kg/m², median (IQR) & 210 & $21.2(19.40-23.40)$ \\
\hline Married, n (\%) & 210 & $69(32.86)$ \\
\hline Education, n (\%) & 210 & \\
\hline Middle school or primary school & & $50(23.8 I)$ \\
\hline High school & & $54(25.7 \mathrm{I})$ \\
\hline College & & $106(50.48)$ \\
\hline Smoking, n (\%) & 210 & $36(17.14)$ \\
\hline Drinking, n (\%) & 210 & $39(18.57)$ \\
\hline Analgesic history, n (\%) & 210 & $46(21.90)$ \\
\hline Sedative history, n (\%) & 210 & II (5.24) \\
\hline $\begin{array}{l}\text { History of prior surgery on the non- } \\
\text { operative eye, } n(\%)\end{array}$ & 210 & $16(7.62)$ \\
\hline $\begin{array}{l}\text { History of prior surgery on the } \\
\text { operative eye, } n(\%)\end{array}$ & 210 & $33(|5.7|)$ \\
\hline ASA status, $\mathrm{n}(\%)$ & 210 & \\
\hline I & & $170(80.95)$ \\
\hline $2^{\#}$ & & $40(19.05)$ \\
\hline SAS score, n (\%) & 210 & \\
\hline$<50$ & & $205(97.62)$ \\
\hline $50-59$ & & $5(2.38)$ \\
\hline
\end{tabular}

Note: ${ }^{\#}$ Includes 2 patients with ASA status 3.

Abbreviations: IQR, interquartile range; BMI, body mass index; ASA status, the patient health status according to the American Society of Anesthesiologists; SAS, self-rating anxiety scale.
Table 2 Surgical and Anesthesia Characteristics of Participants $(\mathrm{N}=210)$

\begin{tabular}{|c|c|c|}
\hline Characteristics & $\mathbf{N}$ & Statistics \\
\hline Duration of operation (min), $\mathrm{n}(\%)$ & 210 & \\
\hline$\leq 30$ & & $|5|(7 \mid .90)$ \\
\hline$>30$ & & $59(28.10)$ \\
\hline Duration of anesthesia $(\mathrm{min}), \mathrm{n}(\%)$ & 210 & \\
\hline$\leq 60$ & & $160(76.19)$ \\
\hline$>60$ & & $50(23.8 I)$ \\
\hline Surgical eye, n (\%) & 210 & \\
\hline Monocular surgery & & I54 (73.33) \\
\hline Binocular surgery & & $56(26.67)$ \\
\hline The operative EOMs, n (\%) & 210 & \\
\hline Medial rectus & & $164(78.10)$ \\
\hline Lateral rectus & & $185(88.10)$ \\
\hline Superior rectus & & II (5.24) \\
\hline Inferior rectus & & $7(3.33)$ \\
\hline Superior oblique & & $12(5.7 I)$ \\
\hline Inferior oblique & & $17(8.10)$ \\
\hline Antiemetic use during the operation, $n$ (\%) & 210 & \\
\hline Single drug & & $7(3.33)$ \\
\hline Combined-drug & & $203(96.67)$ \\
\hline Analgesic use during the operation, $\mathrm{n}(\%)$ & 210 & \\
\hline No & & II (5.24) \\
\hline Flurbiprofen axetil & & 71 (33.8I) \\
\hline Nalbuphine & & $126(60.00)$ \\
\hline Flurbiprofen axetil + Nalbuphine & & $2(0.95)$ \\
\hline
\end{tabular}

Abbreviation: EOMs, extraocular muscles.

\section{Postoperative Discomfort}

Of 210 participants, 199 (94.76\%) experienced mild discomfort (NRS $\leq 3$ any time during the first 24 hours after PACU). The percentage of the participants who reported moderate to severe discomfort (NRS $>3$ ) at $0,2,6,10$, and 24 hours after PACU were shown in Figure 1, and the median of NRS score for discomfort was 0 (IQR, $0-0$ ) at each time-point. During the first 24 hours after PACU, 31 (14.76\%), 11 (5.24\%), 60 (28.57\%), 23 (10.95\%) participants suffered from nausea, vomiting, dizziness, and headache, respectively. Furthermore, the incidence of these symptoms within 2 hours after PACU were 51.61\%, $36.36 \%, 60 \%, 30.43 \%$, respectively.

\section{Potential Factors for Discomfort (Nausea, Vomiting, Dizziness, Headache) Development}

Among the potential factors investigated in the univariate analysis, three were associated with postoperative nausea with a $\mathrm{p}$ value $<0.2$ : female sex, sedative history, and the surgery on inferior oblique. They all were included in 


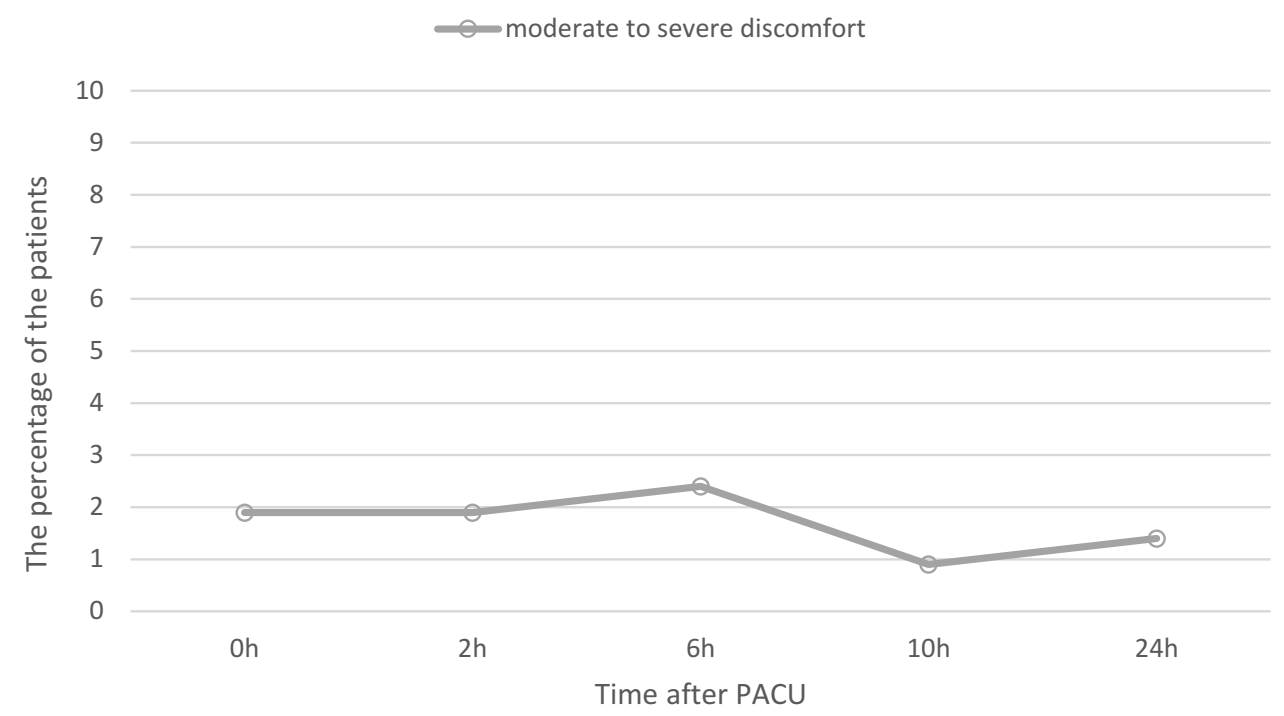

Figure I The percentage of the patients who reported moderate to severe postoperative discomfort at $0,2,6,10$, and 24 hours after PACU. Abbreviation: PACU, postanesthesia care unit.

a multivariable model. However, the other factors, including age, BMI, married, education level, smoking status, drinking status, analgesic history, prior surgery on the operative/non-operative eye, ASA 2 status, mild anxiety, duration of surgery/anesthesia, binocular surgery, the surgery on medial rectus/lateral rectus/superior rectus/inferior rectus/superior oblique, antiemetic and analgesic administration during the operation, did not include in the multivariable model $(p>0.2)$. According to the results of the multivariate analysis, no independent predictor was associated with postoperative nausea ( $p>0.05$, data not shown).

Among the potential factors investigated in the univariate analysis, five were associated with postoperative vomiting with a $\mathrm{p}$ value $<0.2$ : female sex, BMI, mild anxiety (SAS score 50-59), the surgery on inferior rectus, and the surgery on inferior oblique (Table 3 ). They all were included in a multivariable model. According to the results of the multivariate analysis, independent predictors of postoperative vomiting were: female sex, the surgery on inferior rectus, and the surgery on inferior oblique (Table 3 ).

Among the potential factors investigated in the univariable logistic regression analysis, six were associated with postoperative dizziness with a $\mathrm{p}$ value $<0.2$ : female, BMI, analgesic history, prior surgery on the nonoperative eye, ASA status 2, and mild anxiety (SAS score 50-59) (Table 4). They all were included in the multivariable model. According to the results of multivariable analysis, independent predictor of postoperative dizziness was: mild anxiety (Table 4).
Among the potential factors investigated in the univariate analysis, no factor was associated with postoperative headache with a $\mathrm{p}$ value $<0.2$ (data not shown).

\section{Discussion}

With the major transformation from traditional postoperative hospitalization to ambulatory surgery on an outpatient basis, patient safety and comfort in the postoperative period are of the utmost importance when evaluating outpatient procedures. ${ }^{14}$ The purpose of this observational study was analysis of factors associated with postoperative discomfort after ambulatory strabismus surgery under general anesthesia.

Among various ocular surgeries with general anesthesia, the strabismus surgery is one of the most common surgeries that would result in PONV. There have been attempts to use drugs having an antiemetic effect to reduce the incidence of PONV after strabismus surgery. ${ }^{8,15}$ Our data showed the incidence of nausea was $14.76 \%$, vomiting was $5.24 \%$, which was significantly lower than the previously reported incidence of PONV after strabismus surgery under general anesthesia. ${ }^{8,9}$ This risk could be reduced by various methods: propofol and multiple-antiemetic use. In this study, propofol and a combination of antiemetics, such as dolasetron and dexamethasone, or tropisetron and dexamethasone, were used extensively in cases.

Although the mechanism of PONV after strabismus surgery are not still clearly identified, traction of EOMs, is one of the factors for PONV, which induces the oculoemetic reflex and oculocardiac reflex. ${ }^{16}$ In this study, the independent predictors of postoperative vomiting included 
Table 3 Univariable and Multivariable Logistic Regression Analysis of Detecting Potential Factors of Postoperative Vomiting

\begin{tabular}{|c|c|c|c|c|}
\hline \multirow[t]{2}{*}{ Variables } & \multicolumn{2}{|c|}{ Univariable Analysis } & \multicolumn{2}{|c|}{ Multivariable Model } \\
\hline & OR (95\% Cl) & $\mathbf{P}$ & OR (95\% Cl) & $\mathbf{P}$ \\
\hline Age & $0.96(0.88-1.04)$ & 0.310 & & \\
\hline Female sex & $4.55(0.96-21.58)$ & 0.057 & $\begin{array}{l}7.18 \\
(1.20-43.09)\end{array}$ & $0.031 *$ \\
\hline BMI & $0.82(0.65-1.04)$ & 0.103 & $\begin{array}{l}0.82 \\
(0.64-1.07)\end{array}$ & 0.139 \\
\hline Married & $1.18(0.33-4.17)$ & 0.80 & & \\
\hline $\begin{array}{l}\text { Middle or primary } \\
\text { school }\end{array}$ & Ref & 0.851 & & \\
\hline High school & $0(0)$ & 0.997 & & \\
\hline College & $0.69(0.19-2.48)$ & 0.570 & & \\
\hline Smoking & $0(0)$ & 0.998 & & \\
\hline Drinking & $0.42(0.05-3.4 I)$ & 0.420 & & \\
\hline Analgesic history & $0.78(0.16-3.76)$ & 0.760 & & \\
\hline Sedative history & $1.89(0.22-16.25)$ & 0.562 & & \\
\hline $\begin{array}{l}\text { Prior surgery on } \\
\text { the non-operative } \\
\text { eye }\end{array}$ & $1.23(0.15-10.24)$ & 0.850 & & \\
\hline $\begin{array}{l}\text { Prior surgery on } \\
\text { the operative eye }\end{array}$ & $0.52(0.065-4.22)$ & 0.542 & & \\
\hline ASA status 2 & $0(0)$ & 0.998 & & \\
\hline SAS score $(50 \sim 59)$ & $4.88(0.50-47.74)$ & 0.174 & $\begin{array}{l}4.88 \\
(0.38-62.81)\end{array}$ & 0.224 \\
\hline $\begin{array}{l}\text { Duration of } \\
\text { surgery } \leq 30 \mathrm{~min}\end{array}$ & $1.12(0.29-4.38)$ & 0.867 & & \\
\hline $\begin{array}{l}\text { Duration of } \\
\text { anesthesia } \leq 60 \mathrm{~min}\end{array}$ & $0.14(0.30-6.85)$ & 0.654 & & \\
\hline Binocular surgery & $0(0)$ & 0.997 & & \\
\hline $\begin{array}{l}\text { The surgery on } \\
\text { medial rectus }\end{array}$ & $0.47(0.13-1.68)$ & 0.243 & & \\
\hline $\begin{array}{l}\text { The surgery on } \\
\text { lateral rectus }\end{array}$ & $1.37(0.17-11.19)$ & 0.768 & & \\
\hline $\begin{array}{l}\text { The surgery on } \\
\text { superior rectus }\end{array}$ & $1.89(0.22-16.25)$ & 0.562 & & \\
\hline $\begin{array}{l}\text { The surgery on } \\
\text { inferior rectus }\end{array}$ & $8.62(1.47-50.66)$ & 0.017 & $\begin{array}{l}21.85 \\
(2.36-202.48)\end{array}$ & $0.007 *$ \\
\hline $\begin{array}{l}\text { The surgery on } \\
\text { superior oblique }\end{array}$ & $0(0)$ & 0.999 & & \\
\hline $\begin{array}{l}\text { The surgery on } \\
\text { inferior oblique }\end{array}$ & $5.00(1.18-20.79)$ & 0.029 & $\begin{array}{l}9.92 \\
(1.90-51.84)\end{array}$ & $0.007 *$ \\
\hline Multiple antiemetic & $92553228.62(0)$ & 0.999 & & \\
\hline No analgesic & Ref & 0.670 & & \\
\hline Nalbuphine & $126428402.7(0)$ & 0.999 & & \\
\hline Flurbiprofen axetil & $46825334.33(0)$ & 0.999 & & \\
\hline $\begin{array}{l}\text { Flurbiprofen axetil } \\
+ \text { Nalbuphine }\end{array}$ & $1.0(0)$ & 1.000 & & \\
\hline
\end{tabular}

Notes: Boldface indicates $P<0.2 ; * P<0.05$.

Abbreviations: OR, odds ratio; $95 \% \mathrm{Cl}, 95 \%$ confidence intervals; $\mathrm{BMI}$, body mass index; ASA status, the patient health status according to the American Society of Anesthesiologists; SAS, self-rating anxiety scale.

the surgery on inferior rectus and the surgery on inferior oblique, which was similar to the research on previous ophthalmic surgery. ${ }^{17}$ The explain may be the probability
Table 4 Univariable and Multivariable Logistic Regression Analysis of Detecting Potential Factors of Postoperative Dizziness

\begin{tabular}{|c|c|c|c|c|}
\hline \multirow[t]{2}{*}{ Variables } & \multicolumn{2}{|c|}{ Univariable Analysis } & \multicolumn{2}{|c|}{ Multivariable Model } \\
\hline & OR $(95 \% \mathrm{Cl})$ & $\mathbf{P}$ & OR $(95 \% \mathrm{Cl})$ & $\mathbf{P}$ \\
\hline Age & $1.00(0.96-1.03)$ & 0.865 & & \\
\hline Female sex & $1.54(0.84-2.81)$ & 0.160 & $1.57(0.82-3.01)$ & 0.171 \\
\hline BMI & $1.06(0.98-1.14)$ & 0.161 & $1.07(0.98-1.16)$ & 0.125 \\
\hline Married & $0.80(0.42-1.54)$ & 0.509 & & \\
\hline $\begin{array}{l}\text { Middle or primary } \\
\text { school }\end{array}$ & Ref & 0.924 & & \\
\hline High school & $1.16(0.48-2.80)$ & 0.75 & & \\
\hline College & $1.16(0.54-2.53)$ & 0.702 & & \\
\hline Smoking & $1.09(0.50-2.38)$ & 0.827 & & \\
\hline Drinking & $0.81(0.37-1.79)$ & 0.604 & & \\
\hline Analgesic history & $2.04(1.03-4.05)$ & 0.040 & $1.72(0.83-3.58)$ & 0.147 \\
\hline Sedative history & $2.13(0.62-7.25)$ & 0.227 & & \\
\hline $\begin{array}{l}\text { Prior surgery on the } \\
\text { non-operative eye }\end{array}$ & $2.02(0.72-5.69)$ & 0.185 & $1.88(0.64-5.54)$ & 0.252 \\
\hline $\begin{array}{l}\text { Prior surgery on } \\
\text { the operative eye }\end{array}$ & $0.90(0.39-2.07)$ & 0.807 & & \\
\hline ASA status 2 & $1.85(0.90-3.80)$ & 0.093 & $1.56(0.73-3.35)$ & 0.253 \\
\hline SAS score (50 59) & $10.39(1.14-94.91)$ & 0.038 & $10.22(1.10-95.35)$ & $0.041 *$ \\
\hline $\begin{array}{l}\text { Duration of } \\
\text { surgery } \leq 30 \mathrm{~min}\end{array}$ & $1.41(0.72-2.78)$ & 0.317 & & \\
\hline $\begin{array}{l}\text { Duration of } \\
\text { anesthesia } \leq 60 \mathrm{~min}\end{array}$ & $1.07(0.53-2.16)$ & 0.852 & & \\
\hline Binocular surgery & $1.53(0.80-2.94)$ & 0.201 & & \\
\hline $\begin{array}{l}\text { The surgery on } \\
\text { medial rectus }\end{array}$ & $1.21(0.58-2.53)$ & 0.617 & & \\
\hline $\begin{array}{l}\text { The surgery on } \\
\text { lateral rectus }\end{array}$ & $0.85(0.35-2.10)$ & 0.729 & & \\
\hline $\begin{array}{l}\text { The surgery on } \\
\text { superior rectus }\end{array}$ & $0.91(0.23-3.56)$ & 0.894 & & \\
\hline $\begin{array}{l}\text { The surgery on } \\
\text { inferior rectus }\end{array}$ & $1.88(0.41-8.64)$ & 0.420 & & \\
\hline $\begin{array}{l}\text { The surgery on } \\
\text { superior oblique }\end{array}$ & $0.81(0.21-3.08)$ & 0.751 & & \\
\hline $\begin{array}{l}\text { The surgery on } \\
\text { inferior oblique }\end{array}$ & $1.37(0.48-3.88)$ & 0.555 & & \\
\hline $\begin{array}{l}\text { Multiple } \\
\text { antiemetic }\end{array}$ & $1.02(0.19-5.43)$ & 0.977 & & \\
\hline No analgesic & Ref & 0.602 & & \\
\hline Nalbuphine & $1.21(0.3 \mid-4.66)$ & 0.783 & & \\
\hline Flurbiprofen axetil & $1.81(0.46-7.19)$ & 0.398 & & \\
\hline $\begin{array}{l}\text { Flurbiprofen axetil } \\
+ \text { Nalbuphine }\end{array}$ & $0.00(0.00)$ & 0.999 & & \\
\hline
\end{tabular}

Notes: Boldface indicates $P<0.2 ; * P<0.05$.

Abbreviations: OR, odds ratio; $95 \% \mathrm{Cl}, 95 \%$ confidence intervals; $\mathrm{BMI}$, body mass index; ASA status, the patient health status according to the American Society of Anesthesiologists; SAS, self-rating anxiety scale.

of PONV increases as traction on the EOMs increases, thus less accessible muscles (such as the inferior oblique and inferior rectus), are more likely to result in PONV after surgery. ${ }^{17}$ In the future, more studies are needed to 
confirm the potential mechanisms about this result. Furthermore, our study showed that female sex was strongly associated with postoperative vomiting, which has been demonstrated a well-known independent risk factor for PONV in many studies. ${ }^{18,19}$

For a safe discharge, patients must be able to walk without dizziness. This study showed $28.57 \%$ patients reported dizziness during the first 24 hours after surgery. The high incidence of dizziness might be explained by the residual effect of anesthetics as well as inadequate hydration. $^{20}$ Moreover, surgical outcome, for example, change of eye position postoperatively might be attributed to the dizziness symptom, and visual recovery training after the surgery could help to relieve dizziness. ${ }^{21}$ Based on the multivariate analysis performed within this study, the independent predictor of postoperative dizziness was mild anxiety. Thus, a multimodal approach, which could include the identification of patients with anxiety, and optimizing perioperative management of these patients, such as minimizing anesthetics and adequate hydration, would be of greater significance.

Within the first 24 hours after PACU, our study showed minority of patients had postoperative nausea, vomiting, dizziness, and headache, but $94.76 \%$ patients experienced mild discomfort, and the median of NRS for discomfort was 0 (IQR, $0-0)$ at $0,2,6,10$, and 24 hours after PACU. This suggests that ambulatory strabismus surgery is safe and comfortable, if performed by an experienced strabismus surgeon, with optimized perioperative management.

One limitation of our study is lack of generalizability to other surgical procedures or other surgical populations. Moreover, this study design is observational. In the future, larger cohort studies are needed to confirm these results. In addition, another study, on factors associated with postoperative discomfort in children undergoing ambulatory strabismus surgery, is encouraged. Additionally, postoperative discomfort is an individual and widely variable experience that involves psychosocial, surgical, and environmental factors, ${ }^{22}$ and attempts to predict discomfort should be mindful of the limitations in doing so.

In conclusion, patients undergoing ambulatory strabismus surgery tended to experience postoperative nausea and dizziness. Independent risk factors for postoperative vomiting were female sex, the surgery on inferior rectus, and the surgery on inferior oblique. Meanwhile, the independent risk factor for postoperative dizziness was mild anxiety. Awareness about these factors may help health workers to improve postoperative discomfort management in ambulatory strabismus surgery.

\section{Data Sharing Statement}

We intend to share individual deidentified participant data that underlie the results reported in this article, after deidentification (text, tables, figures). Meanwhile, study protocol will be available. All data included in this study are available upon request by contact with the corresponding author. Moreover, the data will be available immediately following publication. No end date.

\section{Acknowledgments}

This study was supported by the clinical research project of Zhongshan Ophthalmic Center of Sun Yat-sen University (3030901010073). Jingxia Huang and Jing Lin are co-first authors for this study.

\section{Author Contributions}

All authors contributed to data analysis, drafting or revising the article, gave final approval of the version to be published and agreed to be accountable for all aspects of the work.

\section{Disclosure}

The authors report no conflicts of interest in this work.

\section{References}

1. Tang H, Dong A, Yan L. Day surgery versus overnight stay laparoscopic cholecystectomy: a systematic review and meta-analysis. Dig Liver Dis. 2015;47(7):556-561. doi:10.1016/j.dld.2015.04.007

2. Cullen KA, Hall MJ, Golosinskiy A. Ambulatory surgery in the United States, 2006. Natl Health Stat Rep. 2009;11:1-25.

3. Fortier J, Chung F, Su J. Unanticipated admission after ambulatory surgery-a prospective study. Can J Anaesth. 1998;45(7):612-619. doi:10.1007/BF03012088

4. Shen YD, Chen CY, Wu CH, Cherng YG, Tam KW. Dexamethasone, ondansetron, and their combination and postoperative nausea and vomiting in children undergoing strabismus surgery: a meta-analysis of randomized controlled trials. Paediatr Anaesth. 2014;24 (5):490-498. doi:10.1111/pan.12374

5. Sadhasivam S, Shende D, Madan R. Prophylactic ondansetron in prevention of postoperative nausea and vomiting following pediatric strabismus surgery: a dose-response study. Anesthesiology. 2000;92 (4):1035-1042. doi:10.1097/00000542-200004000-00021

6. Kaufman S, Martin-Sheridan D. Postoperative nausea and vomiting after strabismus surgery: mechanisms, treatment, and implications for practice. CRNA. 1996;7(3):139-143.

7. Ye H, Chen R, Lian X, et al. Risk factors associated with postoperative pain and discomfort in oculoplastic surgery with general anesthesia: a prospective study. J Pain Res. 2018;11:407-415. doi:10.2147/JPR. S156104

8. Price DD, Bush FM, Long S, Harkins SW. A comparison of pain measurement characteristics of mechanical visual analogue and simple numerical rating scales. Pain. 1994;56(2):217-226. doi:10.1016/03043959(94)90097-3 
9. Korn BS, Kikkawa DO, Vasani SN, et al. Evaluation of patient comfort with outpatient orbital surgery. Orbit. 2007;26(1):19-22. doi:10.1080/01676830600972708

10. Henzler D, Kramer R, Steinhorst UH, Piepenbrock S, Rossaint R, Kuhlen R. Factors independently associated with increased risk of pain development after ophthalmic surgery. Eur $J$ Anaesthesiol. 2004;21(2):101-106. doi:10.1097/00003643-200402000-00004

11. Samakouri M, Bouhos G, Kadoglou M, Giantzelidou A, Tsolaki K, Livaditis M. [Standardization of the Greek version of Zung's Selfrating Anxiety Scale (SAS)]. [Article in Greek, Modern] Psychiatriki. 2012;23(3):212-220.

12. Dunstan DA, Scott N, Todd AK. Screening for anxiety and depression: reassessing the utility of the Zung scales. BMC Psychiatry. 2017;17(1):329. doi:10.1186/s12888-017-1489-6

13. All W. Diagnostic and Statistical Manual of Mental Disorders. Washington, DC, USA: American Psychiatric Association; 1995.

14. Shi Y, Weingarten TN, Mantilla CB, Hooten WM, Warner DO. Smoking and pain: pathophysiology and clinical implications. Anesthesiology. 2010;113(4):977-992. doi:10.1097/ALN.0b0 $13 \mathrm{e} 3181 \mathrm{ebdaf9}$

15. Behman R, Cleary S, McHardy P, et al. Predictors of post-operative pain and opioid consumption in patients undergoing liver surgery. World J Surg. 2019;43(10):2579-2586. doi:10.1007/s00268-01905050-7
16. Kenny GN. Risk factors for postoperative nausea and vomiting. Anaesthesia. 1994;49(Suppl):6-10. doi:10.1111/j.1365-2044.1994. tb03576.x

17. Lerman J. Surgical and patient factors involved in postoperative nausea and vomiting. Br J Anaesth. 1992;69(7 Suppl 1):24S-32S. doi:10.1093/bja/69.supplement 1.24S

18. Son J, Yoon H. Factors affecting postoperative nausea and vomiting in surgical patients. $J$ Perianesth Nurs. 2018;33(4):461-470. doi:10.1016/j.jopan.2016.02.012

19. Yi MS, Kang H, Kim MK, et al. Relationship between the incidence and risk factors of postoperative nausea and vomiting in patients with intravenous patient-controlled analgesia. Asian J Surg. 2018;41 (4):301-306. doi:10.1016/j.asjsur.2017.01.005

20. Ip HY, Abrishami A, Peng PW, Wong J, Chung F. Predictors of postoperative pain and analgesic consumption: a qualitative systematic review. Anesthesiology. 2009;111(3):657-677. doi:10.1097/ALN.0b013e3181aae87a

21. Jackson J, Castleberry C, Galli M, Arnoldi KA. Cerebral palsy for the pediatric eye care team part ii: diagnosis and treatment of ocular motor deficits. Am Orthopt J. 2006;56:86-96. doi:10.3368/aoj.56.1.86

22. Raeder J, Gupta A, Pedersen FM. Recovery characteristics of sevoflurane- or propofol-based anaesthesia for day-care surgery. Acta Anaesthesiol Scand. 1997;41(8):988-994. doi:10.1111/j.13996576.1997.tb04825.x

\section{Publish your work in this journal}

The Journal of Pain Research is an international, peer reviewed, open access, online journal that welcomes laboratory and clinical findings in the fields of pain research and the prevention and management of pain. Original research, reviews, symposium reports, hypothesis formation and commentaries are all considered for publication. The manuscript management system is completely online and includes a very quick and fair peer-review system, which is all easy to use. Visit http:// www.dovepress.com/testimonials.php to read real quotes from published authors. 